\title{
Tératozoospermie monomorphe et altérations nucléaires
}

\author{
Nathalie RIVES \\ Laboratoire de Biologie de la Reproduction - CECOS, Centre d'Investigation Clinique Inserm 0204, \\ CHU Hôpitaux de Rouen, Rouen
}

La formation du spermatozoïde est l'aboutissement d'un processus qui associe des divisions cellulaires et une maturation cellulaire complexe intéressant le noyau, le cytoplasme et la membrane plasmique au cours de la spermiogenèse. Des dysfonctionnements de la spermiogenèse conduit à l'élaboration de spermatozoïdes anormaux sur le plan morphologique ou fonctionnel. Un sperme est considéré comme "normal" s'il possède au moins $30 \%$ de spermatozoïdes à morphologie normale. Les spermằtozoïdes humains présentent un fort pourcentage d'anomalies morphologiques les différenciant de ceux des autres espèces. On note cependant une certaine constance du profil de la tératozoospermie chez un même individu d'un recueil de sperme à l'autre.

Dans les années 50 , les études des pionniers de la spermiologie comme Mac Leod ont montré l'importance de la morphologie des spermatozoïdes dans l'évaluation de la fertilité. L'avènement de l'assistance médicale à la procréation confirme l'importance du pourcentage de formes normales dans l'éjaculat. Cependant, l'association de nombreuses anomalies morphologiques aggrave le handicap fonctionnel du spermatozoïde et altère son pouvoir fécondant. II en est de même lorsque les spermatozoïdes sont porteurs d'une anomalie morphologique dite spécifique retrouvée sur la majorité des spermatozoïdes d'un éjaculat. Dans ces situations, la microscopie électronique est un complément indispensable afin d'établir le diagnostic de cette anomalie morphologique spécifique.

Les altérations morphologiques des spermatozoïdes sont le plus souvent polymorphes et plus exceptionnel- lement monomorphes (macrocéphalie, microcéphalie, globozoospermie et dyskinésie flagellaire). L'existence d'une relation a été suggérée entre les tératozoospermies monomorphes et les anomalies chromosomiques spermatiques ou aneuploïdie, ceci est particulièrement vérifié pour les spermatozoïdes macrocéphales mais plus difficilement pour les autres altérations morphologiques monomorphes. Ainsi, on peut supposer que des perturbations de la méiose puissent retentir non seulement sur le contenu chromosomique du spermatozoïde mais aussi sur son niveau de maturité nucléaire et cytoplasmique.

L'évaluation de l'aneuploïdie des spermatozoïdes s'effectue habituellement par hybridation in situ en fluorescence (FISH). Cependant, les techniques conventionnelles de FISH ne permettent pas d'établir une relation spécifique entre le phénotype du spermatozoïde et son contenu nucléaire. La combinaison de la FISH et d'un marqueur spécifique du flagelle ou de l'acrosome permet d'associer certaines altérations morphologiques du spermatozoïde (flagelles courts, absents ou irréguliers - acrosomes absents ou irréguliers - tête dupliquée, tête mutinucléée, tête macrocéphale) au contenu chromosomique.

\section{Correspondance :}

Dr Nathalie RIVES - Laboratoire de Biologie de la Reproduction - CECOS, Centre d'Investigation Clinique Inserm 0204, CHU Hôpitaux de Rouen, 76031, Rouen -

Tel 0232888225 - Fax 0235982007 -

Email nathalie.rives@chu-rouen.fr 
De ces études, nous avons pu ainsi constater qu'un aspect morphologique normal du flagelle du spermatozoïde ne constituait pas un bon critère de sélection d'un spermatozoïde au contenu chromosomique normal dans les dyskinésies flagellaires. En revanche, chez les hommes fertiles aux paramètres spermatiques normaux, une relation semble exister entre spermatozoïdes à flagelles anormaux et aneuploïdie spermatique.

En ce qui concerne la microcéphalie ou les altérations morphologiques de l'acrosome, l'absence d'acrosome ou une morphologie anormale de l'acrosome est un facteur prédictif d'un contenu chromosomique anormal. Dans les tératozoospermies associant spermatozoïdes multinucléés, multiflagellés, têtes multiples et/ou macrocéphales, il est possible d'identifier une population variable de spermatozoïdes à tête unique et au contenu chromosomique normal.

Dans ces différents types de tératozoospermie, seront aussi envisagés la fréquence des défauts de condensation de l'ADN spermatique ainsi que les défauts de fragmentation de l'ADN. En effet, les anomalies chromosomiques ne sont probablement pas les seules anomalies du noyau spermatique dans le cadre des tératozoospermies monomorphes. Ainsi, des défauts de condensation nucléaire voire une fragmentation anormale de l'ADN peuvent s'associer à l'aneuploïde.

Cette évaluation à différents niveaux du noyau du spermatozoïde peut être utilisée avant la prise en charge en Assistance Médicale à la Procréation (AMP) ou en cas d'échecs répétés des techniques d'AMP lorsque la tératozoospermie a été initialement mal identifiée ou sous estimée, afin de décider la poursuite ou l'arrêt de la prise en charge.

Manuscrit reçu : septembre 2007 ; accepté septembre 2007.

Communication au XXVlème Congrès de la SALF, décembre 2007, Colmar 